\title{
A Rare Case of a Thoracic Spinal Hemangiopericytoma
}

\section{Derou Keableon Louis ${ }^{1 *}$, Irié Bi Gohi Serge², Tokpa André1, Konan Serge Yao1, Bougaci Nassim³, Lonjon Michel $^{3}$}

\author{
${ }^{1}$ Neurosurgery Department, Bouake Teaching Hospital, Bouake, Ivory Coast \\ ${ }^{2}$ Anesthesia-Intensive Care Unit, Bouake Teaching Hospital, Bouake, Ivory Coast \\ ${ }^{3}$ Neurosurgery Department, Pasteur Hospital, Nice, France \\ Email: *keableon1@gmail.com
}

How to cite this paper: Louis, D.K., Serge, I.B.G., André, T., Yao, K.S., Nassim, B. and Michel, L. (2019) A Rare Case of a Thoracic Spinal Hemangiopericytoma. Open Journal of Modern Neurosurgery, 9, 1-6. https://doi.org/10.4236/ojmn.2019.91001

Received: November 14, 2018

Accepted: December 4, 2018

Published: December 7, 2018

Copyright $\odot 2019$ by authors and Scientific Research Publishing Inc. This work is licensed under the Creative Commons Attribution International License (CC BY 4.0).

http://creativecommons.org/licenses/by/4.0/

\begin{abstract}
Spinal hemangiopericytomas are rare tumors. To date, only 80 cases of spinal hemangiopericytomas have been reported in the literature. The postoperative evolution of this condition in rare cases can be complicated by a symptomatic compressive epidural hematoma that can be the source of serious neurological complications requiring emergency surgery. We report a rare case of intradural and extramedullary spinal hemangiopericytomas with favorable evolution after treatment in an 82 years old woman.
\end{abstract}

\section{Keywords}

Hemangiopericytoma, Spine, Intradural, Extramedullary, Radiotherapy, Emergency, Surgery

\section{Introduction}

The hemangiopericytoma is a rare and malignant tumor of the central nervous system classically developed from the meninges and able to metastasize outside the neuraxis [1]. It represents less than $1 \%$ of all the central nervous system tumors and $2 \%$ of the primary meningeal tumors [2]. Hemangiopericytomas have been first considered to be angioblastic meningiomas before OMS classified them as non-meningothelial tumors of mesenchymal origin in 1993 [1]. Since the 2016 central nervous tumors classification of the WHO, they are grouped with solitary fibrous tumors (TFS) under the same entity: TFS/hemangiopericytoma [3]. Most primary hemangiopericytomas of the central nervous system are intracranial and rarely located in the spine. The secondary spinal location is most often related to an intracranial origin [4]. The diagnosis is histologic and mole- 
cular [2]. The complete surgical resection remains the standard treatment and the interest of radiotherapy remains controversial [5]. The outcome in case of an incomplete surgical resection is generally marked by a high rate of the tumor relapse in the local site [6]. To date, only about 80 cases of spinal hemangiopericytomas have been reported in the literature [1]. We report a rare case of an intradural and extra-medullary thoracic hemangiopericytoma.

\section{Case Report}

An 82-year-old patient was hospitalized for a motor deficit of the lower right limb that was disturbing her gait and evolving for three weeks. The clinical examination of this patient showed a deficit in the right thigh and in the levator muscle of the right foot respectively at $3 / 5$ and $2 / 5$. There were no ascending fibers disorders. MRI of the thoracic spine revealed anintradural and extramedullary T9 - T10 tumor with a homogenous enhancement after gadolinium injection (Figure 1). The diagnosis of meningioma and neuroma was evoked on the basis of the characteristics of the tumor on MRI.A Surgical resection was performed. This surgical procedure consisted of a laminectomy of T9 and an almost complete tumor resection with a capsular residue which represented approximately $10 \%$ of the tumor's size. This capsular residue was very adherent to the spinal cord. The anatomopathological diagnosis of the lesion was Grade III hemangiopericytoma according to the WHO classification (Figure 2). The tumor exhibited a typical histological appearance of hemangiopericytoma with nuclear positivity of the STAT6 marker in immunohistochemistry. Grade III was proposed because of a high mitotic index. The immediate postoperative follow-up was marked by a compressive epidural hematoma of the operative site which worsened the motor deficit of the patient who passed to $0 / 5$. This hematoma was evacuated in emergency with none complication during the immediate post operative follow-up. The patient's discharge was allowed a few days later with a beginning of recovery of the motor strength regarding the right lower

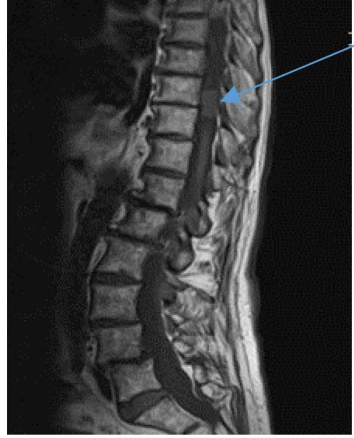

(a)

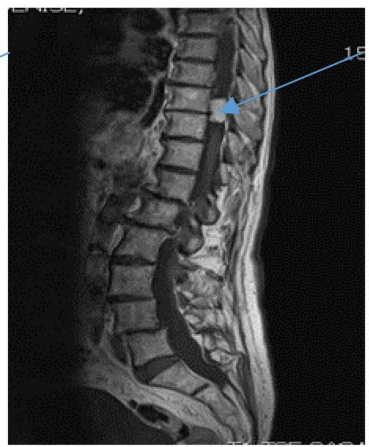

(b)

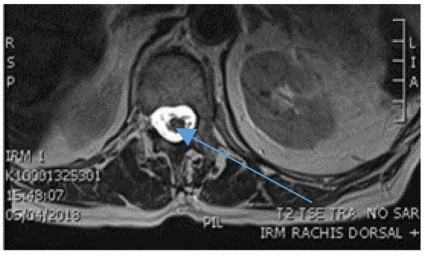

(c)

Figure 1. MRI sequence T1 saggital sections ((a) and (b)) and T2 axial section (c). (a) and (b): without and with injection showing an intradural and extramedullary tumor with homogeneous enhancement after injection of gadolinium, (c): Intradural and extramedullary lesion. Blues arrows show the tumor. 


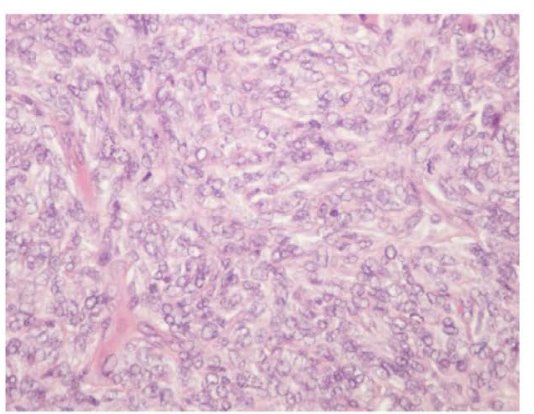

(a)

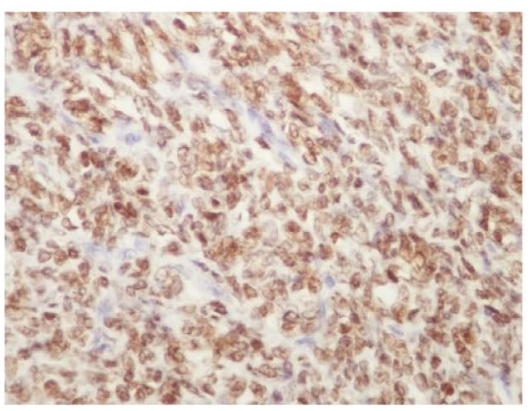

(b)

Figure 2. (a) Histological section showing a proliferation of monomorphic cells irrigated by many capillaries and encompassed in a thin fibrous network; (b): histological section after anti-STAT6 immunohistochemical staining, $\times 400$ : tumor nuclei positivity for STAT6, confirming the diagnosis of hemangiopericytoma

$\operatorname{limb}(3 / 5$ proximal and $1 / 5$ distal). A functional reeducation prescription has been made and is still going on. A brain MRI performed in order to the research for a primary tumor site was negative. A complementary radiotherapy, more precisely a volumetric arc therapy with intensity modulation has been carried out. It has consisted of a 52 grays administered in 26 fractions. At the postoperative consultation five months after the surgery the patient had an improvement in motor strength. This one was rate at $3 / 5$. The research for distant metastatic foci after radiotherapy was negative.

\section{Discussion}

Hemangiopericytomas are rare vascular and aggressive tumors developed at the expense of Zimmermann's pericytes [1] [7]. The 2016 WHO classification considers hemangiopericytomas and solitary fibrotic tumors of the nervous system as the same pathological entity due to immunohistochemical and molecular lesional similarities [3]. The primary spinal location of hemangiopericytomas has been rarely reported in the literature [2]. To date, about 80 cases of spinal hemangiopericytomas have been reported and the cervico-dorsal spine is the most frequent site [1]. The clinical manifestation can be summarized as a motor deficit, radiculalgia and sensory disorders [1]. The sphincter disorders are relatively rare and late [8]. The case we report was clinically manifested only by a deficit in the right lower limb. The radiological figures of spinal hemangiopericytomas are nonspecific and have similarities with other spinal tumors, including meningiomas and neuromas [5]. The tumor, most often, has an intradural, extramedullary localization, well circumscribed with a dural attachment [1]. Purely intramedullary or purely intradural locations are rare [8]. The tumor is most often heterogeneously enhanced on MRI in T1 sequence with injection of gadolinium [9]. The presence of voids signals zones in T2 sequence is indicative of the hypervascularized nature of the tumor [5]. The case we report had rather a homogeneous enhancement with $\mathrm{T} 1$ contrast MRI and no T2 void signal. The differential radiological diagnosis of spinal hemangiopericytomas is mainly dis- 
cussed with meningiomas, schwannomas, neurofibromas and neuroblastomas [2]. The lack of specificity of the clinical and radiological manifestations makes the diagnosis unclear. The diagnosis of certainty is histomolecular: it is based on the association of a characteristic morphology and a nuclear staining of the STAT6 protein in immunohistochemistry. This nuclear staining reflects the presence of the translocation of the STAT6 gene, characteristic of the TFS/hemangiopericytoma entity [3]. Because of their rarity, there is no standard protocol for the treatment of spinal hemangiopericytomas. Most therapeutic modalities are based on reported clinical cases or therapeutic modalities that have been successful for intracranial hemangiopericytomas [7]. Thus, surgery is the first-line treatment of spinal hemangiopericytomas [8]. Complete resection of the tumor is the main objective. The extent of tumor resection correlates with a low rate of long-term tumor recurrence [10]. However, the vascular nature of the tumor may be responsible for significant intraoperative bleeding thus making complete excision difficult. This haemorrhagic risk is thought to be responsible for high mortality and morbidity. Preoperative tumor endovascular embolization has been shown to be effective in reducing bleeding during the surgery and facilitating tumor resection [11]. In our case, the tumor could not be resected completely because of the capsular residue adhering to the spinal cord. The high risk of recurrence of spinal hemangiopericytomas, especially in cases of incomplete resection and high grade tumor, which was the case for our patient, justifies the use of adjuvant radiotherapy [12]. Adjuvant radiotherapy would promote local control of the tumor [1]. For some authors, the combination of complete tumor resection with radiotherapy would significantly increase the time of tumor recurrence compared to exclusive tumor resection for high-grade tumors (213 months versus 43 months) [13]. Exclusive radiotherapy would be useful in case of nonoperable or small tumor [12]. Chemotherapy alone has not yet been shown to be effective in the treatment of spinal hemangiopericytomas [12]. But anti-angiogenic molecules could produce better results and are still under study [5]. The recurrence time for hemangiopericytomas in general is between 2 and 18 years on average, warranting a close monitoring, especially during the first five years after surgery [5].

Spinal Hemangiopericytomas are rare tumors which are usually difficult to be totally removed.

The relapse of the tumors locally or metastases appearance is very common regarding the outcome of this disease. A close post-operative monitoring could be useful to detect such complications.

\section{Conclusion}

Spinal hemangiopericytomas are rare tumors. The diagnosis is relatively difficult because of the rarity of the tumor and the nonspecific nature of the clinico-radiological manifestations. Complete surgical resection is the optimal treatment. Adjuvant radiotherapy should be given in the case of a high grade 
tumor and incomplete tumor resection. Close monitoring would detect local recurrence and possible metastases.

\section{Conflicts of Interest}

The authors do not declare any conflict of interest.

\section{References}

[1] Das, A., Singh, P.K., Suri, V., Sable, M.N. and Sharma, B.S. (2015) Spinal Hemangiopericytoma: An Institutional Experience and Review of Literature. European Spine Journal, 24, 606-613. https://doi.org/10.1007/s00586-015-3789-1

[2] Chew, L.S., Han, X.J., Tan, K.K. and Bundele, M.M. (2017) Hemangiopericytoma of the Thoracic Spine: A Case Report. Journal of Surgical Case Reports, 2017, No. 7. https://doi.org/10.1093/jscr/rjx121

[3] Louis, D.N., Perry, A., Reifenberger, G., von Deimling, A., Figarella-Branger, D., Cavenee, W.K., Ohgaki, H., Wiestler, O.D., Kleihues, P. and Ellison, D.W. (2016) The 2016 World Health Organization Classification of Tumors of the Central Nervous System: A Summary. Acta Neuropathologica (Berlin), 131, 803-820. https://doi.org/10.1007/s00401-016-1545-1

[4] Lee, J.K., Kim, S.H., Joo, S.P., Kim, T.S., Jung, S., Kim, J.H. and Lee, J.H. (2006) Spinal Metastasis from Cranial Meningeal Hemangiopericytomas. Acta Neuropathologica (Wien), 148, 787-790. https://doi.org/10.1007/s00701-006-0766-9

[5] Shirzadi, A., Drazin, D., Gates, M., et al. (2013) Surgical Management of Primary Spinal Hemangiopericytomas: An Institutional Case Series and Review of the Literature. European Spine Journal, 22, S450-S459.

https://doi.org/10.1007/s00586-012-2626-Z

[6] Ramakrishna, R., Rostomily, R., Sekhar, L., Rockhill, J. and Ferreira, M. (2014) Hemangiopericytoma: Radical Resection Remains the Cornerstone of Therapy. Journal of Clinical Neuroscience, 21, 612-615. https://doi.org/10.1016/j.jocn.2013.08.006

[7] Kaur, J., Pandit, S., Sharma, M.C., Julka, P.K. and Rath, G.K. (2015) Intradural Extra Medullary Hemangiopericytoma of Dorsal Spine. Child's Nervous System, 31, 173-175. https://doi.org/10.1007/s00381-014-2505-5

[8] Liu, H., Yang, A., Chen, N., Yang, J., Qiu, X. and Zhang, J. (2013) Hemangiopericytomas in the Spine: Clinical Features, Classification, Treatment, and Long-Term Follow-Up in 26 Patients. Neurosurgery, 72, 16-24.

https://doi.org/10.1227/NEU.0b013e3182752f50

[9] Ackerman, P.D., Khaldi, A. and Shea, J.F. (2011) Intradural Hemangiopericytoma of the Thoracic Spine: A Case Report. The Spine Journal, 11, e9-e14. https://doi.org/10.1016/j.spinee.2011.04.030

[10] Türk, C.Ç., Kara, N.N., Süren, D., Özdöl, Ç., Gediz, T. and Yildiz, S. (2015) Distinctive Characteristic Features of Intramedullary Hemangiopericytomas. Asian Spine Journal, 9, 522-528. https://doi.org/10.4184/asj.2015.9.4.522

[11] Zhang, P., Hu, J. and Zhou, D. (2013) Hemangiopericytoma of Cervicothoracic Spine: A Case Report and Literature Review. Turkish Neurosurgery. https://doi.org/10.5137/1019-5149.JTN.8299-13.1

[12] Jia, Q., Zhou, Z., Zhang, D., et al. (2018) Surgical Management of Spinal Solitary Fibrous Tumor/Hemangiopericytoma: A Case Series of 20 Patients. European Spine Journal, 27, 891-901. https://doi.org/10.1007/s00586-017-5376-0 
[13] Schiariti, M., Goetz, P., El-Maghraby, H., Tailor, J. and Kitchen, N. (2011) Hemangiopericytoma: Long-Term Outcome Revisited. Clinical Article. Journal of Neurosurgery, 114, 747-755. https://doi.org/10.3171/2010.6.JNS091660 